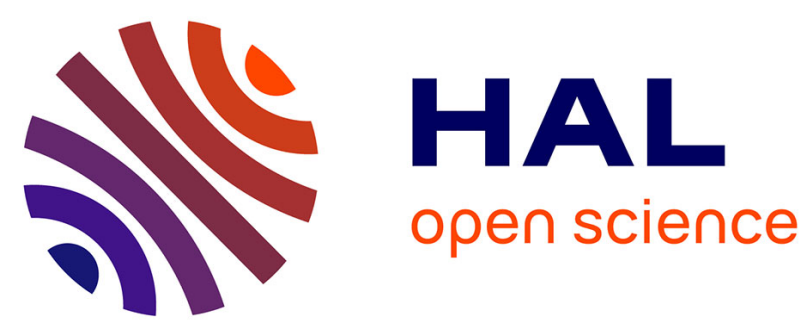

\title{
Big Data et conception d'un système d'information d'aide à la décision clinique: vers une gestion sociocognicielle de la responsabilité médicale?
}

Christine Sybord

\section{- To cite this version:}

Christine Sybord. Big Data et conception d'un système d'information d'aide à la décision clinique : vers une gestion sociocognicielle de la responsabilité médicale?. Les Cahiers du numérique, 2016, Enjeux du big data et identification des données médicales 12 (1-2), pp.73-108. hal-01560622

\section{HAL Id: hal-01560622 \\ https://hal.science/hal-01560622}

Submitted on 11 Jul 2017

HAL is a multi-disciplinary open access archive for the deposit and dissemination of scientific research documents, whether they are published or not. The documents may come from teaching and research institutions in France or abroad, or from public or private research centers.
L'archive ouverte pluridisciplinaire HAL, est destinée au dépôt et à la diffusion de documents scientifiques de niveau recherche, publiés ou non, émanant des établissements d'enseignement et de recherche français ou étrangers, des laboratoires publics ou privés.

\section{다(1)(2)}

Distributed under a Creative Commons Attribution - ShareAlikel 4.0 International 


\title{
Big Data et conception d'un système d'information d'aide à la décision clinique: vers une gestion sociocognicielle de la responsabilité médicale?
}

\author{
Christine SYBORD
}

\begin{abstract}
Résumé
Avec les défis de stockage, d'analyse, de protection des données de santé à caractère personnel posés par le Big Data, la question de l'évaluation d'un Système (d'information) d'Aide à la Décision Médicale (SADM) devient cruciale autant pour le médecin que pour le patient. Dans ces conditions, l'article aborde la conception d'un SADM en questionnant ses conditions d'intégration dans les pratiques médicales de la décision clinique. La première partie, après avoir caractérisé le Big data sur un plan économique, présente le cadre cognitif et technique des SADM. Le cadre et le statut d'un SADM étant posés, la deuxième partie analyse les fondements juridiques du triptyque patient - médecin - SADM/Big Data. Cette analyse conduit à une remise en question strictement physique ou morale de la responsabilité médicale à l'avantage d'une réflexion sur les conditions épistémologiques et éthiques de la gestion d'une responsabilité médicale étendue. La troisième partie présente ainsi une analyse critique des systèmes de connaissances (Ermine, 1996), en référence à la Théorie du Système Général (Le Moigne, 1994). Cette analyse critique amène le cadrage théorique de la conception systémique sociocognicielle d'un SADM. Cette conception permet une gestion éthique de la responsabilité médicale étendue et facilite ainsi la communication médecin-patient. Le cadrage théorique étant posé, la quatrième partie présente le modèle sociocogniciel d'un SADM en référence à une approche sociocognitive. Le modèle instrumente l'organisation des relations (médecin, patient, SADM) qui se co-construisent et interviennent dans la prise de décision clinique.
\end{abstract}

\section{Mots-clés}

Big Data, Décision clinique, Système d'Aide à la Décision Médicale, Responsabilité médicale, Modélisation des systèmes complexes, Systèmes de connaissances, Approche socio-cognitive.

\section{Introduction}

Le terme de «Big Data» a été évoqué pour la première fois à la fin des années 2000, par le cabinet d'études Gartner Group, pour désigner la profusion d'informations générées par les usages d'Internet, de la téléphonie mobile, des réseaux sociaux. Aujourd'hui, Il est utilisé dans la quasi-totalité des domaines d'activité, dont celui de la santé. Dans ce contexte d'infobésité médicale, les utilisateurs partagent des données personnelles, voire intimes, sur leur vie. Ce partage génère des risques dont celui de profilage des individus. Nouvel objet de l'économie du numérique, le Big Data représente ainsi un thème d'actualité qui consiste à se demander : comment gérer cette énorme quantité de traces numériques qui sont autant d'indices renseignant les pratiques des acteurs connectés aux Technologies de l'Information et la Communication (TIC) Internet?

Pour appréhender cette question, deux perspectives sont possibles :

- La première est d'ordre logistique informationnelle et consiste à conserver les informations pour un éventuel traitement, ce qui revient à s'interroger sur les manières de stocker ces données brutes ;

- La deuxième est d'ordre cognitif et consiste à analyser de manière la plus rapide possible ces données nombreuses et hétérogènes pour permettre aux acteurs impliqués dans la sphère économique, de prendre des décisions informées en temps réel. En effet, ne parait-il pas bien inutile d'accumuler des données si on ne sait pas les traiter?

Finalement, au-delà de l'engouement actuel, l'économie actuelle du numérique, évoquée très souvent avec le Big data, rencontre beaucoup de problématiques anciennes dont le questionnement de fond porte sur les conditions éthiques, sociales, techniques, économiques, cognitives du partage des informations qui, depuis l'informatisation des années 60, sont devenues de plus en plus nombreuses et variées. 
Dans le champ de la santé, ces questions anciennes ont été débattues dès les années 70 par le parlement lors de la mise en place du «fichier national de santé des français ». Ce fichier avait pour but de répondre à des besoins socio-économiques d'optimisation des comptes de l'assurance maladie et de prise en compte du patient. Son utilisation interrogeait déjà les conditions d'accès au fichier, les conditions d'hébergement et de sécurisation des données de santé par nature sensibles, la(es) place(s) et prérogative(s) réelle(s) du patient et des professionnels de santé dans ce dispositif socio-informatique (appelé quelques années plus tard système d'information médical).

Avec le phénomène «Big Data » actuel, le questionnement des conditions d'usages des TIC est de nouveau d'actualité, notamment sous l'angle, en sciences humaines et sociales, de la surveillance des individus connectés aux divers réseaux et dispositifs Internet (Ramonet, 2015). En France, comme les données de santé à caractère personnel sont juridiquement considérées comme des données sensibles, leur collecte ou leur traitement informatique sont encadrés par plusieurs textes réglementaires et dans de nombreux cas, interdits sans autorisation spéciale. Ce cadre législatif existe depuis 1978 avec la loi dite Informatique et libertés. Le cadre de l'activité d'hébergement de données de santé à caractère personnel est fixé par l'article L. 1111-8 du code de la sante publique inséré par la loi n 2002-303 du 4 mars 2002, dite « Loi Kouchner ». Malgré cette législation sur la protection des données nominatives, les technologies du Big Data fondées sur le Web sémantique permettent aujourd'hui une fouille très poussée des bases de données, ce qui engendre des risques psychosociaux dont les conséquences peuvent être très graves (perte d'emploi, marginalisation, etc.) (Colloc, 2015).

Dans cette perspective des conditions d'usages des TIC Big Data, cet article interroge, d'un point de vue sociocognitif et d'un point de vue juridique, les conditions d'intégration d'un Big Data dans les pratiques médicales et notamment lors de la prise de décision clinique.

L'intégration d'un Big Data dans le champ clinique est en effet délicate à plusieurs titres :

- Premièrement, la décision clinique est un processus complexe, constitué de différentes étapes cognitives qui interagissent (Banning, 2006), (Sybord, 2000) ;

- Deuxièmement, les technologies Big Data brouillent de nouveau les frontières des échanges patientmédecin (Thoër, 2013), ces échanges ayant déjà été bousculés avec l'introduction de l'informatique médicale dans les années 70 ;

- Troisièmement, la qualité des informations recueillies sur Internet par les soignants ou les soignés est très inégale (Eysenbach et al., 2002), (Hargrave et al., 2003) ;

- Quatrièmement, les principes éthiques associés au droit de l'information médicale sont remis en question notamment avec les objets connectés (Zolynski, 2015).

En sciences de gestion, le Big Data correspond à un «nouveau mouvement d'innovation socio-technique qui participe à réorganiser les activités de l'offre et de la demande» (Vayre, 2013). Ce mouvement d'innovation, caractérisé par la multitude et la fluidité, interpelle toutes les organisations qui doivent repenser, sans délai, leurs modèles d'affaires, leurs stratégies, leurs métiers et leurs missions (Martinet, 2014), (Colin et Verdier, 2015). Face à ce défi stratégique, le système d'information (SI) défini par Rowe (2007, p171) comme "un système d'interprétation d'un ensemble d'acteurs sociaux qui, dans un contexte organisationnel finalisé et récursif, mémorisent et transforment des représentations via des technologies de l'information et des modes opératoires » est un outil de gestion (Chiapello et Gilbert, 2013) qui peut, dans le contexte de décision clinique, instrumentaliser cette réorganisation de l'offre et de la demande.

Plus précisément, le type de SI en adéquation avec le Big Data est décisionnel car le SI de stockage et de compréhension d'une multitude de flux informationnels doit fournir des voies d'actions originales au praticien soignant (cf. les deux perspectives énoncées ci-dessus). Utilisant largement les TIC, ces systèmes, appelés "Systèmes Décisionnels » et abrégés SAD (Systèmes d'Aide à la Décision) sont détaillés dans Lebraty (2006). Les SAD représentent l'ensemble des SI qui servent de support aux activités de pilotage des organisations, et en particulier à la prise de décision.

En médecine clinique, ces SI sont appelés des Systèmes d'Aide à la Décision Médicale (SADM) et sont définis comme «des applications informatiques dont le but est de fournir aux cliniciens en temps et lieux utiles les informations décrivant la situation clinique d'un patient ainsi que les connaissances appropriées à cette situation, correctement filtrées et présentées afin d'améliorer la qualité des soins et la santé des patients » (Berner, 2009). Constitué de nombreuses informations et connaissances variées, le SADM est un système complexe, 
multidimensionnel, basé sur la cognition des médecins et des patients qui ont des droits et des devoirs (Sybord, 2000).

En pratique, l'impact de ces SADM sur les situations de décision individuelles et collectives, qui sont complexes et risquées (Le Moigne, 1974), (Falque et Bougon, 2009), (Fernandez, 2013), ont toujours un impact mitigé sur la prise de décision clinique et leur intégration pratique est faible (Comble et al., 2010), (Flottorp et al., 2013), (Darmoni et al., 2014). Ce constat est corroboré, selon la revue de la littérature des SI en gestion, dans la synthèse de Vidal et Lacroux (2000) et par Le Moigne et Amabile (2006), Gillet et Gillet (2013), Sybord (2013).

Comment alors expliquer cet écart entre un intérêt scientifique avéré et un désintérêt pratique résistant ?

Interroger l'écart entre l'intérêt scientifique d'un SADM et son désintérêt pratique résistant revient à évaluer la manière dont le SADM a été appréhendé et élaboré. Autrement dit, analyser cet écart consiste à questionner le processus de conception et d'élaboration d'un SADM, aujourd'hui largement conditionné par le phénomène Big Data. En outre, avec les défis de stockage, d'analyse, de protection des données de santé à caractère personnel posés par le Big Data, cette question de la conception d'un SADM devient cruciale autant pour le médecin que pour le patient (Gallard, 2014).

Pour aborder cette conception d'un SADM, nous procédons méthodologiquement par étapes. Chaque étape constitue une partie.

La première partie, après avoir caractérisé le Big data sur un plan économique, présente le cadre cognitif et technique des SADM. L'explicitation des différentes étapes cognitives du processus de la décision clinique permet d'appréhender la place effective d'un SADM lors de l'exercice de la décision clinique : le statut d'un SADM est alors défini, ce qui permet de passer à la deuxième étape. La deuxième partie analyse les fondements juridiques du triptyque patient - médecin - SADM/Big Data. Cette analyse conduit à une remise en question strictement physique ou morale de la responsabilité médicale lors de l'utilisation d'un Big Data, à l'avantage d'une réflexion sur les conditions épistémologiques et éthiques de la gestion d'une responsabilité médicale étendue. Cette réflexion constitue la troisième étape. La troisième partie présente, en effet, une analyse critique des systèmes de connaissances (Ermine, 1996), en référence à la Théorie du Système Général (Le Moigne, 1994). Cette analyse critique amène le cadrage théorique de la conception systémique sociocognicielle d'un SADM. Cette conception permet une gestion éthique de la responsabilité médicale étendue et facilite ainsi la communication médecin-patient. Ce cadrage théorique conduit à la quatrième et dernière étape de la méthodologie conceptuelle. La quatrième partie présente le modèle SADM qui s'appuie sur cette approche sociocognicielle. Le modèle instrumente l'organisation des relations (médecin, patient, SADM) qui se coconstruisent et interviennent dans la prise de décision clinique.

\section{Big Data et décision clinique : quel statut pour le système d'information d'aide à la décision ?}

Le Big Data ne fait pas référence uniquement aux « données », mais également aux technologies de l'information qui permettent, notamment, une analyse sémantique de ces données. Cette analyse en profondeur aide le médecin dans la pratique complexe que constitue la décision clinique. C'est pourquoi, après avoir caractérisé le Big data sur un plan socio-économique, nous expliciterons les différentes étapes cognitives de la décision clinique qui «incarne» le Dossier Médical Informatisé (DMI), pour ensuite aborder les outils informatiques d'aide à la décision clinique.

\subsection{Les 5 «V » du Big data (Mart, 2015)}

Comme il a été indiqué précédemment, la définition du Big Data n'existe pas car le Big data désigne plutôt un phénomène, touchant tous les domaines, qui s'enrichit au cours du temps avec des enjeux de plus en plus pertinents. De plus, les données sont internes ou externes à l'organisation, sachant que les données les plus intéressantes, pour une organisation, sont désormais celles qui résident hors du SI en place (Intel, 2012). En effet, les évolutions technologiques récentes permettent aussi aux consommateurs de générer plus de données dans des temps beaucoup plus courts. Concrètement, on ne travaille plus que sur des données structurées mais aussi sur des données brutes et disparates qui viennent de sources très variées, à très grande vitesse, puisque le volume de données augmente désormais de manière exponentielle. 
Du point de vue d'un utilisateur qui a à gérer cette masse de données, structurées ou non structurées, le traitement des Big Data est ainsi conditionné par la règle des $5 \mathrm{~V}$ (initialement les $3 \mathrm{~V}$ ) :

- Volume, qui désigne la quantité d'informations à stocker et à analyser ;

- Vitesse, qui désigne les délais d'actualisation, de visualisation et d'analyse (en temps réel ou quasi réel, voire analyse en streaming sans stockage);

- Variété, qui désigne les sources et les formats de données structurées et non structurées (images, textes, tweets, traces numériques, contenu multimédia).

Cette multitude et fluidité des données implique de contrôler l'exactitude des contenus, d'évaluer des rumeurs ou diffusions malveillantes (sur les réseaux sociaux notamment), de repérer un capteur défectueux, etc. Ce contrôle introduit le quatrième « $V$ » et la caractéristique de Véracité d'une donnée.

C'est précisément sur ces 4 premières caractéristiques et en particulier sur la caractéristique de Vitesse que de nombreux systèmes d'analyse, de CRM, de personnalisation, de point de vente ou autres, échouent encore à ce jour car ils peuvent seulement traiter les données par lots toutes les quelques heures, dans le meilleur des cas. Or, ces données n’ont alors déjà plus vraiment de valeur puisque le cycle de génération de nouvelles données a déjà commencé.

Ce souci de la valeur de la donnée introduit alors le cinquième « $\mathrm{V} »$ : Valeur

Conformément à ce qui a déjà été mentionné, le Big Data se caractérise aussi par des données externes, non structurées issues d'Internet, des réseaux sociaux, de capteurs ou de logs. Ces données majoritairement générées par les patients via le net, permettent de retracer, par exemple, leurs interactions avec une maladie : les soignés ont maintenant la parole produisant ainsi de plus en plus d'informations non structurées et aussi utiles à la conception d'un SADM.

Pour l'organisation, un des défis du Big Data est finalement qu'il ne suffit pas de simplement "gérer" les données, mais de les exploiter activement, de façon à ce qu'elles aient un impact économique concret sur sa performance globale. Par exemple, Catalina Marketing a mis en place, pour ses clients, une stratégie de coupons de réduction ciblés en analysant en temps réel les tickets de caisse. Cette stratégie a conduit à doubler la rentabilité des campagnes de couponning (Poiraud, 2013). Parallèlement à cet impact économique se pose aussi la question éthique de la surveillance des comportements et des personnes (Murakami Wood et Ball, 2013).

Concernant la décision clinique, le médecin praticien est également concerné par ce phénomène Big Data qui, en médecine, trouve ses origines dans l'informatique médicale (Colloc, 2015). Cette dernière n'a cessé de se développer pour aider les médecins à deux contraintes économiques apparemment contradictoires : d'une part, diminuer le temps décisionnel (cf. Vitesse) pour faire face à une charge de travail toujours plus lourde (cf. Volume et Variété) et d'autre part, augmenter la fiabilité et la validité de la décision (cf. Véracité et Valeur). L'informatique médicale a connu une accélération considérable avec l'avènement d'Internet dans les années 90. Elle connaît aujourd'hui une nouvelle évolution avec les connaissances du patient devenu actif et de mieux en mieux informé avec les technologies d'exploitation automatique. A ce titre, les connaissances du patient participent aussi au processus cognitif de la décision clinique.

\subsection{Le processus cognitif de la décision clinique}

La pratique du médecin est ainsi enrichie par des moyens informatiques d'investigation, d'acquisition et de stockage de données épidémiologiques et cliniques. Les données épidémiologiques sont issues d'études épidémiologiques qui analysent les facteurs influant sur la santé et les maladies de population. Elles permettent d'enrichir le corpus de connaissances d'un grand nombre de spécialités médicales, ce qui contribue ainsi à améliorer les pratiques cliniques et prévenir l'émergence de pathologies. Les données cliniques sont, elles, recueillies lors de l'exercice clinique qui consiste à observer et écouter l'histoire clinique de chaque patient pour ensuite mener à bien les différentes étapes cliniques nécessaires à sa prise en charge. La décision clinique est une « histoire de relations humaines » (Colloc, 2015, figure 7). D’un type de maladie à l'autre, ces différentes étapes peuvent prendre des formes différentes mais représentent, dans leur enchaînement, le processus cognitif qui conduit à la prise de décision clinique.

Le diagnostic est la première étape cognitive. Elle correspond au raisonnement clinique qui conduit à poser le diagnostic et décider d'actions thérapeutiques (Pelaccia et al., 2011). Selon G.Lazorthes dans (Guibert, 1992), ce raisonnement est fondé sur la capacité de synthèse d'un ensemble de renseignements fournis par le contact direct 
avec le malade et les investigations complémentaires. Il repose sur un long apprentissage. D'ailleurs, selon Claude Bernard (2013), le sens clinique est basé, d'une part, sur la capacité de faire une récolte des données, «collecte globale non classée, objective » et, d'autre part, sur la capacité de faire une interprétation de ces données en essayant de "trouver dans sa mémoire la liste des maladies dont la combinaison de symptômes théoriques est d'accord avec celle observée chez les malades ». Il est à noter l'importance des connaissances acquises antérieurement grâce aux différentes mémoires : la mémoire sensorielle (perception des signes cliniques), la mémoire à court terme (élaboration des hypothèses de pathologies), et la mémoire à long terme (support de l'expérience médicale) (Sybord et Colloc, 1997).

Il existe deux types de diagnostic.

Le premier est le diagnostic clinique qui recherche les signes cliniques fonctionnels, physiques, les antécédents familiaux et personnels qui manifestent les états pathologiques. Le diagnostic clinique s'appuie sur les sémiologies, médicale et chirurgicale. Il comporte deux phases en miroir avec d'éventuelles itérations :

- le diagnostic positif recherche les signes d'appel permettant de formuler un ensemble d'hypothèses ;

- le diagnostic différentiel recherche les signes discriminants permettant l'élimination d'hypothèses élaborées à l'étape précédente.

Le deuxième est le diagnostic étiologique qui étudie la cause des manifestations pathologiques, c'est à dire les processus qui conduisent à l'apparition et à l'évolution des maladies.

Globalement, on peut dire que le diagnostic clinique se nourrit de connaissances superficielles statiques (heuristiques) tandis que le diagnostic étiologique exploite des connaissances profondes, issues des technologies Big data, capables d'expliciter les causes de l'apparition des maladies et de leur évolution.

Le pronostic est la deuxième étape cognitive. Elle correspond au raisonnement pronostique qui conduit à prévoir les issues plausibles de la maladie diagnostiquée. En effet, le pronostic est un acte au cours duquel le médecin tente d'anticiper l'évolution d'une maladie et son issue probable (guérison, consolidation, issue fatale, etc.). Même si elle peut s'appuyer sur l'épidémiologie, cette prédiction est en pratique toujours difficile à réaliser car elle doit tenir compte des formes cliniques classiques de l'affection, des facteurs individuels du malade (génétiques, psychologiques, physiques), les antécédents cliniques et les chances de réussite de la thérapeutique choisie.

Les connaissances permettant d'élaborer un pronostic proviennent d'abord du diagnostic clinique (cf. première étape cognitive), si possible étiologique, d'une évaluation de l'état général du malade, des éléments de surveillance de l'efficacité du traitement en cours. Les études épidémiologiques, l'expérience du praticien basée sur l'observation de cas similaires, sont des arguments pour établir le pronostic.

Le traitement est la troisième étape cognitive. Elle correspond au raisonnement thérapeutique qui conduit à prescrire au patient un traitement adapté à la maladie diagnostiquée et au pronostic prévu. La décision thérapeutique dépend de critères provenant du diagnostic et du pronostic de la pathologie dont souffre le patient. En outre, sont examinés toutes les connaissances permettant d'évaluer sa capacité à bien supporter le traitement prescrit. A ce titre, sont distingués :

- Les traitements curatifs, dont l'objectif est de guérir totalement ou de consolider l'état du malade : stopper l'évolution de la maladie en prescrivant un antibiotique (dans le cas d'une angine par exemple);

- Les traitements préventifs, dont l'objectif est de prévenir la survenue d'une pathologie parfois grave (par exemple la vaccination);

- Les traitements symptomatologiques sont utilisés lorsque l'on ne dispose pas de diagnostic étiologique et que l'on désire faire cesser des symptômes et des manifestations fonctionnelles désagréables. Ils sont provisoires et à réserver aux affections bénignes ;

- Les traitements palliatifs sont employés lorsque le pronostic vital est compromis, on se contentera de traitements symptomatologiques destinés à soulager à défaut de soigner.

Le praticien commence donc par définir le but de son traitement en s'appuyant sur les antécédents personnels et familiaux qui permettront de déterminer les contre-indications éventuelles (absolues ou relatives), le diagnostic et le pronostic de la pathologie. Ensuite, il précise le but thérapeutique en tenant compte des éventuels traitements déjà en cours. Enfin, il décide d'un traitement, cette décision prenant la forme d'une stratégie thérapeutique composée de la prescription de médicaments, de conduites à tenir et de mesures hygiéno - diététiques sur une période déterminée.

Le suivi thérapeutique est la quatrième étape cognitive. Elle comprend plusieurs aspects complémentaires : 
l'observation de l'évolution de l'état du patient et la recherche de l'apparition d'effets secondaires indésirables, voire de maladies iatrogènes (maladie induite par le traitement). Le premier aspect correspond à une comparaison des évolutions classiques concernant la pathologie diagnostiquée (connaissances concernant le pronostic) avec les variations effectivement constatées chez le patient: amélioration, stagnation ou aggravation. Le second aspect correspond à une tâche de diagnostic, soit des effets secondaires, soit de maladies iatrogènes. Le suivi thérapeutique est donc une tâche de diagnostic spécifique sur laquelle le médecin va asseoir sa décision : modifier le traitement ou l'arrêter.

Les données médicales nominatives recueillies et résultant de cette démarche clinique sont, dans la plupart des cas, mémorisées dans le DMI qui contient aussi les résultats des examens complémentaires (analyses biologiques, radiologiques, psychosociales, autres). Intrinsèquement lié au processus de prise en charge du patient, le DMI contient des données d'origine et de nature différentes (administratives, médicales, paramédicales, sociales, etc) qui sont inférées, recueillies et notées par autant de types d'acteurs impliqués dans la prise en charge du patient. A ce titre, le DMI peut être considéré comme un Big Data.

En synthèse, le processus de décision clinique est un processus complexe constitué de nombreuses interactions entre ces différentes étapes cognitives. Son déroulement dépend d’une logique propre au raisonnement clinique, des informations acquises durant les différentes étapes de celui-ci, des événements qui peuvent se produire de manière plus ou moins prévisibles et modifier l'état clinique du patient qui, comme il a déjà été indiqué, participe de plus en plus à ce processus décisionnel. Cette base cognitive est la clé de voûte conceptuelle des systèmes d'aide à la décision clinique.

\subsection{Les systèmes d'aide à la décision clinique}

Le processus complexe qui représente la décision clinique peut être supporté par des outils, modèles et approches informatiques dont l'ensemble est appelé Systèmes d'Aide à la Décision Médicale (SADM) ${ }^{1}$. Rappelons que le but de ces outils informatiques est «de fournir aux cliniciens en temps et lieux utiles les informations décrivant la situation clinique d'un patient ainsi que les connaissances appropriées à cette situation, correctement filtrées et présentées afin d'améliorer la qualité des soins et la santé des patients. » (Berner, 2009). Très concrètement, le médecin introduit, dans le SADM, les données cliniques recueillies. Le système contenant lui-même une grande quantité de données épidémiologiques, peut alors faire des suggestions/ recommandations qui éclaireront le praticien dans sa démarche. En aucun cas, le système ne prendra la décision à la place du médecin.

Les premiers SADM datent des années 70 et ont été des systèmes experts. Ces systèmes correspondent à un logiciel limité dans un domaine de connaissances et prenant en compte des données incertaines et évolutives sur lesquelles interviennent des cheminements de pensée variables (Sybord, 1992). Mycin, considéré comme le précurseur des systèmes experts médicaux à règles de production, a été construit pour fournir un avis sur le diagnostic et le traitement des maladies infectieuses (Buchanan et Shortliffe, 1984). Internist (Pople et al., 1975), Caduceus (Myers et al., 1982) et QMR (Rennel et Shortliffe, 1987) sont des systèmes d'aide à la décision fondés sur des algorithmes de recherche de correspondance (" pattern matching »).

Ces premiers outils ont connu un relatif succès dans les hôpitaux américains. En France, leur déploiement est resté modeste car les médecins perçoivent encore aujourd'hui de tels outils comme une atteinte à leur indépendance professionnelle et à leur pouvoir de décision. Ils perçoivent essentiellement le rôle automatique de la machine (le contenant), au détriment de son rôle d'assistant (le contenu) (Séroussi et Bouaud, 2014).

Depuis le début des années 90, d'autres approches informatiques ont été proposées : la logique floue, les systèmes de raisonnement par cas (Case-Based Reasoning : CBR), les modèles bayésiens, les réseaux de neurones, les cartes cognitives et les Systèmes Multi-Agents (SMA). Les CBR sont notamment utiles pour l'apprentissage de processus décisionnels car ils permettent le stockage de situations cliniques résolues appelées «cas ", ce qui permet la restitution de cas similaires lorsqu'une nouvelle personne vient consulter (Aamodt et Plaza, 1994). Ils peuvent être utilisés conjointement aux ontologies médicales qui mettent à la disposition des praticiens des thésaurus adaptés à chaque étape clinique (Shen et al., 2014). Les SMA, eux, représentent un vaste domaine allant de l'Intelligence Artificielle Distribuée (Ferber, 1995), (Bousquet et al., 2013) à la vie artificielle (Langton, 1997). Il est à noter qu'entre les deux existent des approches hybrides et intermédiaires.

\footnotetext{
${ }^{1}$ Il existe également des systèmes d'aide à la décision sur papier (SFMG, 1996), qui ne sont pas l'objet de notre article.
} 
En synthèse, les SADM se sont développés au rythme des avancées informatiques de ces 50 dernières années. D'abord centrés sur l'aide au diagnostic, ils ont ensuite évolué pour fournir un support à la décision thérapeutique, parallèlement au développement des RPC (Recommandations pour la Pratique Clinique). Ils constituent ainsi un outil incontournable des progrès scientifiques en médecine et sont devenus, à ce titre, indispensables à l'exercice médical. Dans ces conditions pratiques, le statut des SADM peut être qualifié d'« outil intermédiaire de gestion » qui gère les échanges entre le décideur-soignant et le patient-soigné.

Pour autant et en dépit de l'ordonnance 96-345 du 24 Avril 19962 permettant aux médecins généralistes de s'informatiser, les SADM ont encore aujourd'hui un impact mitigé sur la prise de décision clinique et leur intégration pratique est faible. Les principales raisons sont l'évolution constante des connaissances, les difficultés d'interopérabilité au sein des SI médicaux et les résistances des professionnels (Comble et al., 2010), (Flottorp et al., 2013), (Darmoni et al., 2014).

Comment alors expliquer cet écart entre un intérêt scientifique avéré et un désintérêt pratique résistant? Les raisons sont-elles juridiques?

\section{Dans le triptyque (médecin, patient, $\mathrm{SADM}$ ) : quelles frontières pour la responsabilité médicale ?}

Cette partie a pour but d'analyser, sur un plan juridique, l'intégration des SADM dans les pratiques médicales. C'est son caractère utilitaire, appréhendé comme un « outil intermédiaire de gestion » qui est ici mis en avant. En effet, le médecin peut s'appuyer sur le système pour résoudre ses propres problèmes, le système étant conçu de façon à égaler les savoir-faire d'experts reconnus. En outre, le praticien peut observer le comportement du système dans le but d'une auto-formation. Assistant virtuel du médecin, cet outil intermédiaire de gestion ne pourra, en aucun cas, remplacer le praticien dans la prise de risque de toute décision. Ce qui est conforme aux principes de la déontologie médicale, aux obligations contractuelles et devoirs du médecin.

\subsection{Les principes déontologiques}

Sur un plan juridique, l'utilisation d'un assistant virtuel pose la question de l'indépendance du médecin lors de la prise de décision. Cette indépendance est un principe de la déontologie médicale. Avant de poursuivre, précisons que le code de déontologie médicale en vigueur en France repose sur les articles R.4127-1 à R.4127-112 du code de la Santé Publique. L’article 5 du Code de Déontologie médicale prévoit que « le médecin ne peut aliéner son indépendance professionnelle sous quelque forme que ce soit ».

Le corollaire de cette indépendance est la responsabilité : le médecin est lui-même responsable et ne doit pas accepter une position subordonnée telle que sa liberté de jugement et d'action se trouverait amputée ou orientée. Il ne peut s'abriter derrière des instructions données par autrui. Il aura toujours à juger de ce qu'il doit faire, en toute conscience (Villey, 1982), (Hoerni, 2000). Sur ce point, l'article 69 du Code de déontologie médicale est clair : «l'exercice de la médecine est personnel; chaque médecin est responsable de ses décisions et de ses actes ».

A ce principe s'ajoute celui de la liberté du médecin qui s'exerce tant dans l'élaboration du diagnostic que dans la mise en œuvre d'une stratégie thérapeutique, pronostic et suivi thérapeutique compris. La lecture, a contrario, de l'article L.372 du Code de la santé publique, relatif à l'exercice illégal de la profession de médecin, révèle que le diagnostic peut résulter «d'actes personnels, consultations verbales ou écrites et tout autre procédé quel qu'il soit». Ainsi est-il pertinent, pour la question de la responsabilité, de remarquer que les SADM peuvent être considérés comme des "procédés », au sens de l'article L.372. Combiné à l'article 32 du Code de déontologie qui impose l'utilisation de moyens éprouvés, ce texte donne toute liberté au médecin pour élaborer son diagnostic.

Pour la thérapeutique, c'est l'article 8 du code de déontologie qui encadre cette liberté : «Dans les limites fixées par la loi, le médecin est libre de ses prescriptions qui seront celles qu'il estime les plus appropriées en la circonstance. Il doit, sans négliger son devoir d'assistance morale, limiter ses prescriptions et ses actes à ce qui est nécessaire à la qualité, à la sécurité et à l'efficacité des soins. Il doit tenir compte des avantages, des inconvénients et des conséquences des différentes investigations et thérapeutiques possibles ». Ainsi, en proposant des suggestions de traitement, le système ne lie pas le médecin qui doit, en toute circonstance, fournir des soins adaptés. Ce qui nous conduit aux obligations contractuelles et devoirs du médecin.

\footnotetext{
${ }^{2}$ Ordonnance relative à la maîtrise médicalisée des dépenses de soins
} 


\subsection{Les obligations contractuelles et devoirs du médecin}

Les obligations sont essentiellement issues de la jurisprudence et découlent du contrat tacite qui s'établit entre le patient et le médecin qu'il consulte.

Selon la solution désormais classique de l'arrêt Mercier, le médecin est tenu d'une obligation de moyens et non de résultat, sous réserve dans ce dernier cas de quelques situations particulières. Ainsi, «il se forme entre le médecin et son client un véritable contrat, comportant pour le praticien l'engagement, sinon, évidemment de guérir le malade, du moins de lui donner des soins, non pas quelconques, mais consciencieux, attentifs, et, réserve faite de circonstances exceptionnelles, conformes aux données acquises de la science $»^{3}$.

Depuis cet arrêt, c'est une responsabilité contractuelle qui pèse sur les médecins. Mais ce contrat liant le médecin à son patient n'est pas une banale convention de prestations de services. L'idée est qu'il repose sur un rapport de confiance et consiste, selon la formule de l'avocat général Matter dans l'affaire Mercier, dans l'engagement du praticien de donner « des soins conformes à la conscience et à la science médicale ». En toute circonstance, le praticien doit mettre en œuvre les moyens nécessaires pour soigner son patient, sans pour autant assurer la guérison. En effet, les facteurs d'incertitude qui tiennent tant à l'état de la science qu'à la constitution du patient font que le médecin ne peut garantir la guérison du malade. C'est pourquoi, l'obligation du médecin est une obligation de moyens.

Les devoirs du médecin sont celui de compétence et celui de prudence. L'obligation morale de compétence est évoquée à l'article 32 du Code de déontologie : «Dès lors qu'il a accepté de répondre à une demande, le médecin s'engage à assurer personnellement au patient des soins consciencieux, dévoués et fondés sur les données acquises de la science, en faisant appel, s'il y a lieu, à l'aide de tiers compétents ». Cette disposition est complétée par l'article 33 qui précise que : «le médecin doit toujours élaborer son diagnostic avec le plus grand soin, en y consacrant le temps nécessaire, en s'aidant dans toute la mesure du possible des méthodes scientifiques les mieux adaptés et, s'il y a lieu, de concours appropriés ».

Ces deux articles sont la traduction du devoir de savoir et du devoir d'humilité du médecin. Lorsque le médecin ne s'estime pas assez compétent, il doit savoir se mettre en retrait dans l'intérêt de son patient et doit recourir à l'avis d'un tiers : confrère ou système technique. Il répond ainsi à la règle de l'article 60 du Code de déontologie, son alinéa 1er stipulant que «le médecin doit proposer la consultation d'un confrère dès que les circonstances l'exigent ». Il est opportun de remarquer que, sur ce sujet, l'aide apportée au médecin par un (des) système(s) d'aide à la décision médicale peut être une réponse moderne à l'impératif déontologique posé par cet article.

En ce qui concerne le devoir de prudence, depuis Hippocrate, la pratique médicale est gouvernée par la règle primum non nocere, c'est-à-dire que le médecin ne doit pas nuire à son patient, et implicitement, ne pas aggraver son état initial. Ce que le Code de déontologie exprime avec plusieurs règles.

Ainsi, l'article 40 stipule que «le médecin doit s'interdire, dans les investigations et interventions qu'il pratique comme dans les thérapeutiques qu'il prescrit, de faire courir au patient un risque injustifié ». Concrètement, le médecin doit évaluer les avantages et inconvénients de ses décisions. Le devoir de prudence a donc comme corollaire le principe de proportionnalité de la décision, posé à l'article 8 relatif à la liberté de prescription du médecin, cette liberté trouvant sa limite dans l'exigence de qualité, de sécurité et d'efficacité des soins. L'alinéa 2 de l'article 71 précise que le médecin «ne doit pas exercer sa profession dans des conditions qui puissent compromettre la qualité des soins et des actes médicaux ou la sécurité des personnes examinées ».

En synthèse, cet ensemble d'obligations et devoirs du médecin signifie que le praticien doit tout mettre en œuvre pour assurer la sécurité et le respect du patient, tant dans les moyens d'élaboration du diagnostic, de la thérapeutique que dans l'exécution de l'acte de soins. Quatre thèmes clés se dégagent : confiance, conscience, science et indépendance (Sargos, 1996), en référence au but ultime des " échanges patient-soignant » : l'intérêt du malade (Hoerni, 2008). Ils ne sont pas incompatibles avec l'utilisation d'un SADM. Au contraire, le système peut être une réelle aide lors d'une question posée, à condition que le praticien reste vigilant et garde toujours un sens critique vis à vis des propositions émises par la machine, conformément au cycle de décision éthique mis au point par Nicole et Louis Lery et détaillé dans (Colloc, 2015). Il convient de noter que dans ce cycle, les arguments décisionnels sont basés autant sur le patient que sur son environnement culturel, ce qui justifie une

${ }^{3}$ Droit Civil. 20 Mai 1936, D.P. 1936, I, 88, note E.P. 
approche systémique de la décision (abordée dans la partie suivante).

En pratique, si le médecin utilise mal le système d'aide et cause préjudice au patient, ce dernier peut juridiquement avoir recours. Le praticien doit alors pouvoir répondre de sa décision en s'appuyant sur tous les éléments qui ont permis la prise de décision clinique. Le DMI est un élément essentiel dans ce travail.

\subsection{Les voies d'action du patient en cas d'une erreur dommageable}

Cette partie indique les voies d'action du patient qui serait victime d'une erreur dommageable, lors de l'exécution d'un contrat de soins assisté par ordinateur. Le patient victime d'un dommage dispose, en effet, de quatre terrains d'action contre le médecin utilisant un SADM. Ces terrains d'actions sont liés à la notion de responsabilité. Cette dernière, au sens juridique, définit les règles selon lesquelles chacun (personne physique ou morale) est susceptible de répondre des conséquences dommageables de ses actes ou de ses absentions. Il existe quatre types de responsabilité que nous retrouvons dans les terrains d'action ci-dessous.

Le premier terrain est le terrain contractuel, en référence à la responsabilité civile du médecin fondée sur l'obligation de moyens résultant du contrat qui le lie au patient (cf. partie 2.1.). C'est sur le fondement de l'article 1147 du Code civil que le patient engagera la responsabilité contractuelle du médecin qui aurait commis une faute à l'occasion de l'exécution du contrat de soins. A l'appui de sa demande principale, il pourra accessoirement invoquer les articles 1135 du Code civil sur les suites naturelles du contrat, et 1137 qui prescrit une gestion de bon père de famille.

Il est à noter que si la faute se passe dans l'enceinte d'un hôpital, ce n'est pas le praticien qui est responsable personnellement mais le service public hospitalier, en référence au droit commun de la responsabilité administrative. Cette notion de faute de service se caractérise par un manquement aux obligations du service public (Conseil d'État, 2015). Le juge administratif appréciera l'existence d'une telle faute au cas par cas, en prenant en compte les conditions dans lesquelles l'acte a été ou n'a pas été réalisé, la prévisibilité de l'accident, les difficultés de l'acte de comprendre, le comportement du patient, etc. Pour améliorer la protection du patient, le Conseil d'État, dans un arrêt du 10 Avril 1992, a substitué à la notion de faute lourde celle de faute médicale et a ainsi simplifié les conditions de la mise en cause de la responsabilité de l’hôpital public.

Le deuxième terrain est celui de la responsabilité du médecin sur le plan délictuel : la responsabilité pénale. L'action délictueuse, fondée sur l'article 1382 du code civil, est, par exemple, mise en œuvre lorsqu'il ne s'est pas formé de contrat entre le patient et le médecin (le patient était inconscient ou incapable). Seule la responsabilité civile sera examinée par le juge pénal qui ne connaît pas la notion de contrat, mais uniquement celle de délit. Il est à préciser qu'en matière de responsabilité médicale, la loi du 4 mars 2002 a porté à dix ans, à compter de la consolidation du dommage, le délai dans lequel se prescrivent les actions tendant à mettre en cause la responsabilité des professionnels de santé ou des établissements de santé publics ou privés à l'occasion d'actes de prévention, de diagnostic ou de soins (article L. 1142-28 du code de la santé publique). Sur le plan pratique, le principe de non-cumul des responsabilités, contractuelle et délictuelle, impose au patient de choisir entre ces deux voies d'action.

Le troisième terrain est celui de la responsabilité du fait des choses, en référence à l'article 1384 alinéa 1er du Code civil. Cette responsabilité s'applique dans les cas où un individu engage sa responsabilité délictuelle à la suite d'un préjudice qu'il aurait causé à autrui par le biais d'une chose - c'est-à-dire d'un SADM - dont il aurait eu l'usage, la direction et le contrôle au moment du dommage. Classiquement sont distinguées la garde de la structure et la garde du comportement. En raison de l'immatérialité du contenu du SADM, il peut être considéré que la garde de la structure (c'est-à-dire l'ensemble des sources qui ont permis l'élaboration du SADM) a été conservée par le concepteur de l'outil SADM et que la garde du comportement a été transférée à l'utilisateur médecin. Néanmoins, c'est précisément cette notion d'immatérialité qui induit un doute dans l'applicabilité de l'article 1384 alinéa 1er ne référant que les objets matériels.

Le quatrième et dernier terrain est celui de l'action disciplinaire dont le but est d'infliger une sanction au médecin par ses pairs: responsabilité disciplinaire (qualifiée parfois d'ordinale). Cette dernière a pour objet de sanctionner l'auteur d'un manquement aux obligations professionnelles ou déontologiques. Dans la pratique, cette action est compliquée à mettre en œuvre, dans la mesure où le patient, plaignant non médecin, n'est pas visé par l'article L. 417 du Code de la santé publique. Le patient devra ainsi déposer sa plainte au Conseil départemental qui la transmettra lui-même au Conseil régional de l'Ordre des médecins. En outre, le plaignant, 
considéré comme simple témoin, ne pourra pas avoir accès au dossier, en référence aux règles jurisprudentielles (Haji Safar, 2012).

Face à ces terrains d'action en cas de dommage au patient, force est de conclure que seule la responsabilité du médecin (ou administrative, dans le cadre hospitalier) est juridiquement significative, mais n'est ni alourdie, ni allégée par le système. Autrement dit, même si la jurisprudence statue sur des cas juridiques délicats, le cadre juridique du contrat de soins et les règles classiques de la responsabilité médicale fondée sur le code de déontologie ne tiennent compte ni de «L'attention au monde » (Kessous, 2012), ni des " pouvoirs des réseaux » (Colloc, 2015) et ni de «la gouvernementalité algorithmique » (Rouvroy et Berns, 2013). Pourtant, la pratique clinique est aujourd'hui largement conditionnée par l'évolution de la relation médecin-patient (cf. notamment le concept de patient-expert (Gross et Gagnayre, 2013)) et par les avancées technologiques. La réalité d'Internet peut même laisser croire à un monde sans frontières matérielles et politiques...

En conclusion, le médecin devra toujours utiliser les outils informatiques avec discernement, comme il le fait d'ailleurs avec ses autres sources d'information. Comme il a été vu en partie 1, le SADM ne sera jamais une instance décisionnelle, mais prépare l'analyse qui conduit à la décision prise, in fine, par l'individu. La pratique médicale se fondera toujours sur l'examen clinique rigoureux et la conscience du médecin, éléments essentiels de la confiance que porte le patient à son médecin pour que lui soit fait tout ce qui lui est nécessaire : la relation médecin-patient reste ainsi à la base de l'acte médical, mais est modifiée avec les évolutions en cours - tant sociétales que technologiques (Gallard, 2014) - engendrées par le Big Data. Cette modification remet en question le caractère strictement physique ou moral de la responsabilité médicale lors de l'utilisation d'un Big Data, à l'avantage d'un questionnement sur les conditions de gestion d'une responsabilité médicale étendue.

\section{Gestion éthique du triptyque (médecin, patient, SADM) : Apports et limites des systèmes de connaissances?}

En théorie, rappelons que les projets Big data intentent de rendre l'information compréhensible, utilisable, partageable et ouverte. Ils sont supportés par des outils informatiques qui permettent plus de transversalité et de communication patient-médecin, ainsi qu'une réelle puissance d'analyse pour découvrir des informations jusquelà inconnues, tant en clinique qu'en épidémiologie. Dans ces conditions, les outils Big Data aident à la prise de décision clinique et font partie des SADM. Dans la pratique, l'impact de ces SADM sur la prise de décision clinique reste mitigé, faute d'un cadre éthique effectif (cf. partie 2) et d'un cadre socio-cognitif de la démarche clinique (cf. partie 1).

Afin de tenir compte de ces éléments éthiques et socio-cognitifs, qui sont primordiaux dans les usages d'un SADM, cette partie analyse les apports et les limites des systèmes de connaissances (Ermine, 1996) pour la conception d'un SADM. En introduction de cette analyse, nous présentons les approches théoriques de la prise de décision qui, selon certains auteurs en sciences de gestion (David, 2002), (Lebraty, 2006), influencent de manière significative la conception d'un SAD, a fortiori celle d'un SADM.

\subsection{Les approches théoriques de la prise de décision : quels apports pour la conception d'un SADM?}

Selon une approche historique des théories décisionnelles, 3 grands courants se dégagent (Lebraty, 2006).

Le premier courant, qui a nourri les travaux en recherche opérationnelle, se fonde sur l'application d'un modèle normatif de la décision qui doit permettre de déterminer une solution optimale face à un problème posé. Ce dernier est limité par la détermination d'un champ des possibles, dont le calcul est basé sur des analyses probabilistes. La recherche de la solution optimale est définie selon un critère prédéfini.

Pour faire face aux difficultés inhérentes à l'activité décisionnelle, l'incertitude est probabilisée, ne laissant que très peu de place à l'incertain. De même, les préférences des décideurs sont probabilisées avec comme critère de décision, celui de l'utilité subjective. Autrement dit, il s'agit de trouver des techniques qui permettent de cadrer mathématiquement les modèles d'optimisation. Le SAD est ainsi conçu selon des modèles mathématiques de haut niveau, qui confondent optimisation du modèle et optimisation de la réalité décisionnelle.

Même si cette théorie trouve des applications, notamment dans le domaine de l'aide aux décisions financières, elle est difficilement utilisable dans la conception d'un SADM qui part d'un Big Data caractérisé par les 5 «V». 
Le deuxième courant, issu de la psychologie et des sciences cognitives, remet en question les notions de norme et d'optimisation de la décision, à l'avantage de la notion d'heuristique : un raisonnement est heuristique s'il aboutit à un résultat décisionnel satisfaisant qui ne s'appuie pas forcément sur une théorie éprouvée. L'essentiel est de trouver un moyen efficace d'appréhender des problèmes complexes pour lesquels la démarche algorithmique est inapplicable.

Dans cette perspective, A.Newell et H.A.Simon (1972) et des chercheurs de la Carnegie-Mellon University mettent au point une méthode appelée GPS, General Problem Solving. Cette méthode dont le modèle canonique est : intelligence (identification et définition du problème), conception (création et analyse des solutions), choix et évaluation, est le point de départ d'un très grand nombre de SADM. Elle décrit de manière complète le premier système de traitement humain de l'information et rend analysable la décision.

Cette approche cognitive de la décision a été beaucoup utilisée dans la conception des SADM, notamment pour ses apports dans la construction de systèmes à base de connaissances cliniques et épidémiologiques et dans la prise en compte du style cognitif du décideur. Le concept de style cognitif permet d'analyser la manière avec laquelle un individu traite l'information à sa disposition. Ce concept, bien que discutable dans la manière dont il peut influencer la conception d'un SADM, permet de pointer du doigt l'importance de l'Interface Homme Machine (IHM) dans la conception d'un système informatisé. En effet, l'IHM est influente dans la prise de décision dans la mesure où le SADM se doit aussi d'adapter son design au modèle mental des utilisateurs.

En résumé, cette approche psycho-cognitive de la décision prend en compte l'intelligence de la décision (en prescrivant notamment un processus décisionnel), le style cognitif du décideur et l'importance de l'IHM. Ces dimensions sont cohérentes avec les caractéristiques de Volume et de Vitesse d'un Big Data. En revanche, les caractéristiques de Variété et de Véracité d'un Big Data sont incompatibles avec cette théorie qui ne considère que le contexte individuel de la prise de décision.

Le troisième et dernier courant dit de la «décision en situation ${ }^{4}$ introduit le concept de situation décisionnelle. Dans ce courant, les recherches étudient spécifiquement les décisions prises dans des situations réelles, s'opposant en cela aux décisions prises dans des laboratoires (Kahneman et Klein, 2009). Dans ces conditions spécifiques, l'analyse d'une décision prend en compte le contexte dans lequel la décision est prise. Cette approche méthodologique vise à expliquer comment les décideurs agissent en situation (Klein et al., 1993), (Klein, 1998, 2001), dès lors que le décideur aura reconnu la situation décisionnelle.

Ainsi, contrairement au courant précédent, cette approche étudie conjointement les processus cognitifs et les contextes dans lesquels ils s'exercent. Le contexte est défini comme un ensemble d'éléments, perçus par le décideur et exerçant une contrainte sur une ou plusieurs tâches du processus décisionnel. Il est alors à la fois dépendant de la tâche et du décideur. Le contexte désigne aussi le niveau d'expérience permettant de mettre en œuvre les compétences du décideur dans une situation donnée.

Cette subjectivité du décideur conditionné (contraint?) par le contexte interdit à cette approche d'envisager l'ensemble des processus décisionnels. L'analyse repose sur des objectifs mal définis et évolutifs, des données instables, des logiques contradictoires, un horizon temporel limité, des enjeux importants, de nombreux acteurs, des contraintes organisationnelles, un décideur à haut niveau d'expertise pour la tâche à exécuter. Dans ce cadre d'analyse, la prise de décision ne consiste pas à faire le choix entre plusieurs options mais à mettre en œuvre une solution qui réponde aux besoins de la décision. Sur un plan conceptuel, ces réalités objectivent les liens forts existant entre le processus organisationnel, le processus décisionnel et le processus cognitif, dans le cas d'une situation décisionnelle.

En résumé, cette approche a montré sa solidité théorique et sa capacité à être mise en œuvre dans des processus industriels sensibles. Dans une certaine mesure, elle est également adaptée à notre problématique des conditions d'intégration d'un Big Data dans les pratiques médicales car elle permet une représentation de la situation décisionnelle en lien avec le contexte médical : par exemple, prescrire un traitement thérapeutique (protocole de traitement) pour un patient atteint d'une maladie chronique. En outre, elle tient compte de la caractéristique de Variété d'un Big Data qui évolue en permanence avec son environnement (par exemple, la prise en compte de

\footnotetext{
${ }^{4}$ Ce courant est encore appelé approche naturaliste de la décision et vient de la traduction de l'expression anglaise « Naturalistic Decision Making » (Klein, 1993).
} 
l'évolution clinique de cette maladie chronique pour ce patient). En revanche, elle ne permet pas d'évaluer de manière certaine la Véracité et la Valeur des informations qui sont échangées lors d'une situation clinique, c'est-àdire la qualité de la communication soignant-soigné. Or, la dimension éthique, incontournable dans une situation de décision clinique, oblige à s'interroger sur l'efficacité organisationnelle des SADM.

\subsection{Efficacité organisationnelle d'un SADM et systèmes de connaissances}

En sciences de gestion, la question de l'efficacité organisationnelle a été abordée dès la fin des années 70 avec notamment la Théorie du Système Général, en référence à l'ouvrage de Le Moigne (1977, 1994). Cette théorie appréhende le SI comme un système «acteurs-machines " appartenant lui-même au système "organisation » structuré en 3 sous-systèmes :

- Le système opérant transforme les matières premières (matérielles et immatérielles) en produits finis (matériels et immatériels);

- Le système d'information enregistre, mémorise les opérations (les processus) du système opérant en fonction d'un objectif défini, et les met à disposition du...;

- ... système de pilotage, appelé encore système de décision.

Ce dernier coordonne les informations et les processus (propres à la communauté d'acteurs constituant l'organisation), en utilisant ses capacités cognitives d'auto-organisation, voire de conscience (Le Moigne, 1990, 1993).

Cette conception systémique est cohérente avec l'approche théorique de la décision en situation en proposant une vision intégrée des trois niveaux d'action (opératoire, informationnel, décisionnel). Pour la décision clinique, elle est pertinente car elle rend compte de la complexité du processus décisionnel où de nombreux éléments de décision, issus de multiples acteurs, sont en interaction. L'analyse systémique porte sur les interactions entre composants plutôt qu'à l'isolement des composants. Se fondant sur la simulation des systèmes complexes (Le Moigne, 1990, 1993), cette approche systémique a été complétée, au milieu des années 90, par le système «patrimoine de connaissances » qui définit les systèmes de connaissances mis au point par (Ermine, 1996).

Cette approche systémique des connaissances est utile pour notre recherche sur la conception d'un SADM car elle tient compte du caractère irréversible, opératoire et auto-organisant du SADM à la base de l'exercice médical. En effet, l'organisation «ne s'entend que dans le temps, le temps irréversible de l'action» (Le Moigne, 1993, p.76). Pour la décision clinique qui consiste à prescrire en temps réel un traitement adapté à la situation clinique et en adéquation avec les besoins du patient, cette organisation organisante et organisée est illustrée par les différentes étapes cognitives explicitées partie 1.2. De plus, les travaux en psychologie montrent que l'étude des interactions (par exemple, la relation médecin-patient via Internet) implique une conception plus complexe des SADM. Cette conception est complexe car elle doit tenir compte d'une double contrainte : la contrainte intersubjective de la relation patient-médecin et la contrainte inter-objective des circonstances et des transactions de la situation clinique. Cette complexité, inhérent à la conception d'un SADM permet de penser les phénomènes cognitifs en adéquation avec la situation décisionnelle et les acteurs sociaux, ces derniers étant engagés dans des activités finalisées et dotés de représentations qui seront progressivement partagées (Olry-Louis, 2011). Enfin, les situations d'interaction Homme/Machine, propres aux SADM, sont caractérisées par le fait que les individus doivent résoudre des problèmes complexes où se tissent, de manière paradoxale, des liens entre un objet l'ordinateur - et son sujet - respect de la personne - (Michit, 1998), (Callon, 2013). Par exemple, dans le cas d'une décision clinique, le besoin du médecinest de se forger une idée, voire une représentation, aussi exhaustive que possible des environnements (interne et externe) pour que sa prise de décision clinique soit la plus éthique possible.

Les systèmes de connaissances sont définis en référence au « Modèle OIDC » (Opération, Information, Décision, Connaissance), représenté par la figure 1. L'explicitation détaillée de ces systèmes se trouve dans (Ermine et al, 1996).

La connaissance, élément canonique du processus décisionnel, se trouve dans le système «Patrimoine de connaissances ». Ce dernier est défini comme un système actif d'acquisition, de transformation et de transmission de connaissances vers les autres sous-systèmes. Le flux cognitif partant de ce sous-système est appelé flux de cognition : ce dernier correspond à l'appropriation implicite ou explicite du « patrimoine » dans le but de participer au processus de transformation propre au système. Le flux cognitif réciproque s'appelle flux de compétence : ce dernier correspond à la transformation (l'enrichissement ?) des connaissances au cours du temps, 
par le biais des acteurs humains, des ressources matérielles et/ou immatérielles.

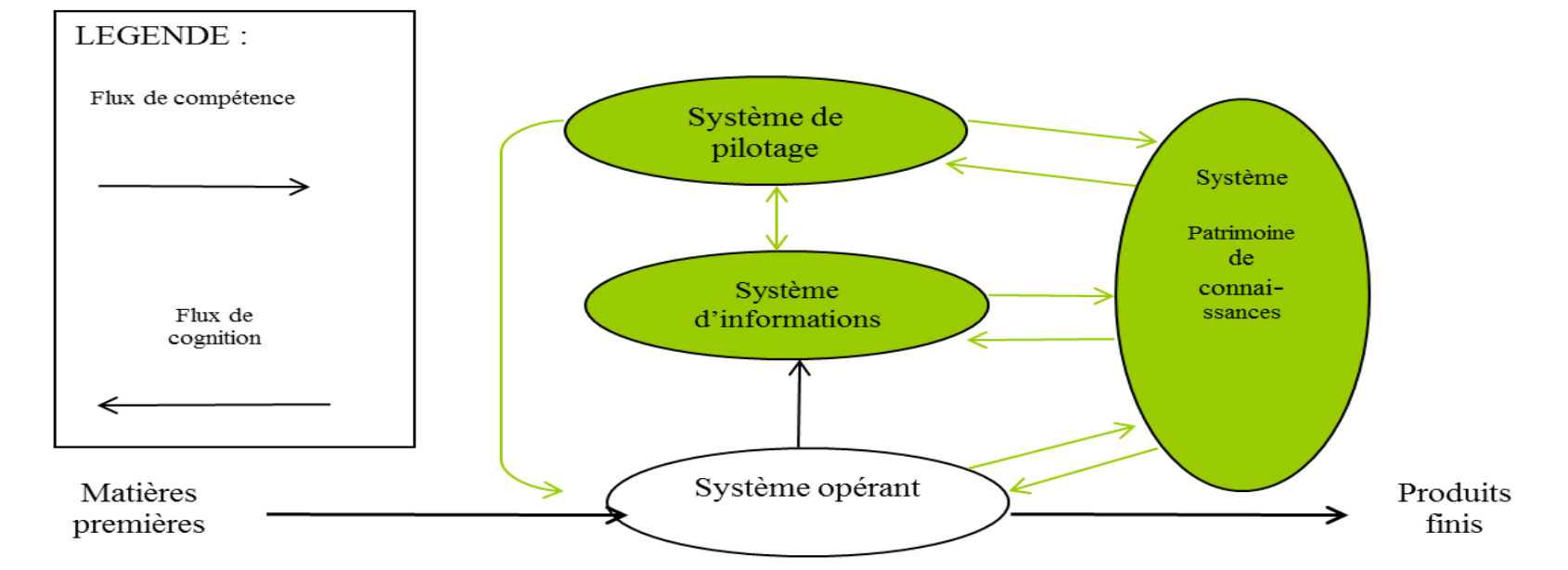

Figure 1 : Le « Modèle OIDC », la base conceptuelle d'un SADM

Cet ensemble (flux, système) correspond à l'espace d'analyse du SADM. Cet espace est spécifié par la couleur verte sur la figure 1. L'avantage d'une telle représentation systémique est la prise en compte globale des 4 dimensions inhérentes à toute pratique de décision clinique (diagnostic, pronostic, traitement, suivi thérapeutique). Les relations et les interactions entre les quatre systèmes confèrent au SADM son caractère « communicant» et organisationnel.

Mais c'est précisément sur ce point que cette représentation systémique et cognitive présente, elle aussi, une limite majeure. En effet, les systèmes (opération, information, décision), hiérarchiquement superposés, génèrent exclusivement des coordinations verticales, ce qui limite, voire empêche, la prise en compte des dynamiques d'apprentissages individuels et collectifs, issues des échanges praticien-patient. Dit autrement, la dimension éthique inhérente à toute décision clinique (cf. partie 2) est négligée.

En conclusion, même si ce modèle systémique OIDC prend en compte les différentes étapes cognitives de la décision clinique il ne permet pas de concevoir un SADM qui respecte les conditions épistémologiques et éthiques d'une responsabilité médicale étendue. Comment alors intégrer le modèle OIDC à la conception d'un SADM qui permette une gestion éthique de la communication médecin-patient ?

\section{Gestion sociocognicielle de la responsabilité médicale : proposition d'un modèle SADM}

Cette partie apporte des éléments de réponse à la question ci-dessus posée, en proposant un modèle qui s'appuie sur une approche socio-cognitive. Cette dernière consiste en l'étude des cognitions sociales, c'est-à-dire des processus par lesquels les individus produisent leurs conduites sociales (Lauriol, 1998). Cette influence réciproque majeure entre domaine cognitif et existence de conduites sociales développées dans une situation d'interaction, est essentielle dans la conception d'un SADM qui accompagne une gestion éthique de la communication médecin-patient.

\subsection{L'approche socio-cognitive}

Lors de l'exercice médical influencé aujourd'hui par le Big data, nous avons vu qu'il existe une influence réciproque majeure entre domaine cognitif, cadre légal et communication médecin-patient. Ces interactions impliquent une conception plus complexe, intersubjective et inter-objective, qui permette de penser les phénomènes cognitifs comme dépendants, dans une certaine mesure, des circonstances et des transactions entre des acteurs sociaux engagés dans des activités finalisées et dotés de représentation qui seront progressivement partagées (Olry-Louis, 2011). 
L'approche socio-cognitive est ainsi pertinente pour les questions que pose la conception d'un SADM qui favorise l'organisation du processus cognitif à la base de la prise de décision clinique. D'une part, la cognition est sociale au sens où les processus de production de connaissances sont intrinsèquement, inévitablement et profondément sociaux (Noucher, 2009). D'autre part, l'organisation est un lieu où des représentations organisationnelles se construisent, où du sens se crée et se transforme sous l'influence des interactions, des conflits cognitifs et des processus d'influence sociale (Ehlinger, 1996).

Autrement dit, pour la conception d'un SADM qui intègre les pratiques médicales dans la décision clinique, les liens indissociables existant entre les processus informationnel, organisationnel, cognitif et décisionnel sont représentés par la figure 2 .

Cette représentation est celle de la figure $1 \mathrm{du}$ « modèle OIDC » qui a été pivoté, sur la droite, d'un angle de $90^{\circ}$, de façon à ce que le système "patrimoine de connaissances » soit la base conceptuelle de l'organisation sociocognitive d'un SADM qui intègre la dimension éthique des usages de l'informatique. Cette base représente, en effet, les multiples savoirs individuels et collectifs qui peuvent intervenir dans le processus cognitif de la décision clinique. Elle favorise la maitrise des processus, ce qui contribue à améliorer la qualité de la décision clinique.

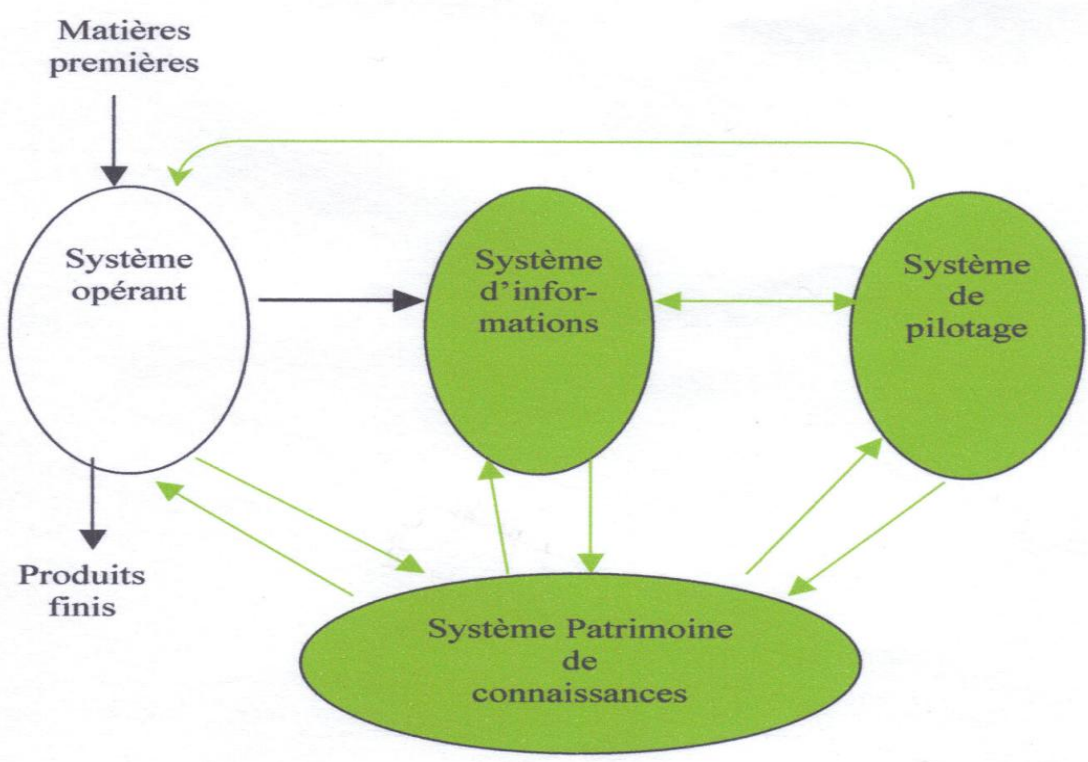

Figure 2 : L'organisation socio-cognitive d'un $S A D M$

Les flux cognitifs et informationnels sont inchangés. En revanche, les liens hiérarchiques classiques, issus du modèle Taylorien, disparaissent à l'avantage de liens transversaux permettant une circulation plus fluide des informations et des connaissances. L'organisation reste ainsi « ouverte » sur les réalités décisionnelles inhérentes à toute pratique clinique.

Dans le cas des activités de prise de décision médicale et en référence au modèle simonien (Simon, 1983), l'objectif de la décision optimale disparaît à l'avantage d'une interrogation sur la nature, voire la légitimité, des compétences disponibles, pour une (des) décision(s) co-construites et de facto satisfaisante(s) pour le patient. Cet ensemble (compétences disponibles, décisions actionnables, responsabilité) est pris en compte dans le modèle présenté ci-dessous.

\subsection{Représentation et explicitation synthétique du modèle SADM cognitivement performant}

La conception d'un SADM étant à la fois technologique et socio-cognitive, nous avons cherché un néologisme qui regroupe à la fois les acquis du génie cognitif et du génie logiciel, ce qui a donné le néologisme :cogniciel. 
Fondé sur l'organisation socio-cognitive d'un SADM ( cf. figure 2), le modèle sociocogniciel est représenté par la figure 3. La représentation circulaire évoque les logiques non linéaires d'apprentissages individuels et collectifs et respecte les liens transversaux de l'organisation socio-cognitive du SADM. Il permet une gestion éthique de la communication médecin-patient: il devient possible de décider au cas par cas quelles données sont confidentielles et quelles sont celles qui peuvent être exploitées.

Conformément aux éléments conceptuels constitutifs d'un SADM :

- Le type de décision, à savoir une décision opérationnelle (poursuite d'un traitement par exemple) ou une décision stratégique (choix d'un traitement) ;

- Le contexte décisionnel, statique et en laboratoire (cf. l'épidémiologie) ou dynamique et en situation réelle (une consultation médicale);

- Le type de connaissance, à savoir connaissances explicites (le symptôme de la fièvre) ou connaissances tacites (la souffrance);

- Les technologies Big data ;

Ce modèle sociocogniciel s'ancre, au sens large, dans une « situation de gestion », qui « se présente lorsque des participants sont réunis et doivent accomplir, dans un temps déterminé, une action collective conduisant à un résultat soumis à un jugement externe » (Girin, 1999). Dans le cas des SADM, le «résultat» est une décision clinique qui intègre de manière éthique tous les savoirs, savoirs-faire, savoirs-être, données et valeurs humaines propres aux pratiques médicales. Dans ces conditions, le médecin et son patient peuvent donner une finalité à leur façon d'échanger et d'agir ensemble.

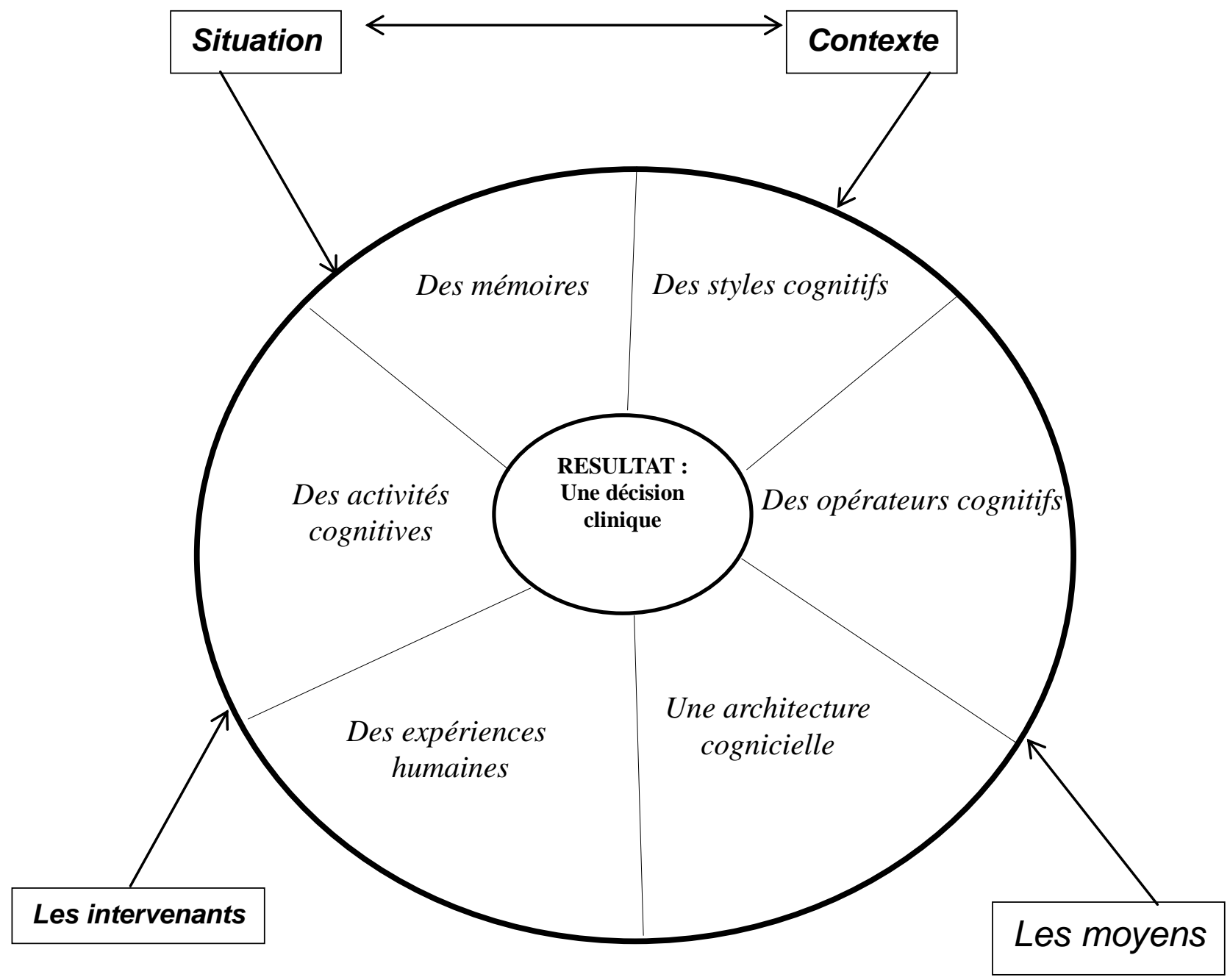

Figure 3: Le modèle socio-cogniciel d'un SADM 
Ce qui est à l'extérieur du cercle correspond aux éléments « environnementaux » de l'activité décisionnelle. Ces éléments rationalisent, voire légitiment la performance de la décision prise. A ce titre, ces éléments environnementaux permettent de lever le doute sur l'applicabilité de l'article 1384 alinéa $1^{\text {er }}$ ne référant que les objets matériels (cf. la partie 3.3.).

L'intérieur du cercle, excepté le "résultat», correspond aux éléments sociocogniciels d'une activité décisionnelle. Ces éléments sont explicités dans (Sybord, 2007). Selon les cas, ces 6 éléments socio-cogniciels peuvent interagir entre eux, via l'architecture cognicielle, et permettent, in fine, une décision partagée par le médecin et son patient.

Afin de rendre plus explicites ces différents éléments, imaginons qu'une personne se rend chez son médecin de famille, en cabinet libéral, pour un mal de tête qui dure depuis 3 jours. Dans ce cas, les éléments « environnementaux » sont:

- Une situation symptomatique de mal de tête qui dure ;

- Un contexte de médecine libérale ;

- Deux intervenants : le médecin et le patient ;

- Les moyens propres à un cabinet de médecine libérale, dont le SADM que le médecin peut utiliser.

Les éléments sociocogniciels sont :

- Les expériences professionnelles du médecin et des expériences personnelles du patient sur les maux de tête persistants ;

- Les activités cognitives qui représentent la manière dont le médecin traite le mal de tête persistant et ce, en fonction de la manière dont le patient supporte, soulage, endure son mal de tête ;

- Les mémoires du médecin, du patient et du SADM représentent l'ensemble des souvenirs en rapport avec les maux de tête déjà rencontrés et tracés ;

- Les styles cognitifs sont les manières d'apprendre du médecin et du patient sur le mal de tête persistant ;

- Les opérateurs cognitifs sont des moyens mnémotechniques ou stratégiques de traiter la douleur occasionnée par le mal de tête persistant et ce, en lien avec les autres éléments sociocognitifs ;

- L'architecture cognicielle est une sorte de « cerveau » du modèle.

Cette dernière est définie à partir du paradigme SMA (Systèmes Multi-Agents). Elle est détaillée dans (Colloc et Sybord, 2003). L'architecture SMA métabolise la dimension collective de la pratique de la décision. Son moteur est une boîte à outils qui suit les innovations techniques comme par exemple l'exploitation automatique de données non sollicitées et non structurées d'un Big Data. Il convient de noter ici que ces nouvelles logiques d'exploitation et d'exploration de données sont maitrisées et encadrées par le modèle sociocogniciel qui permet une protection organisationnelle et une sécurité des données personnelles (cf. la partie 3.).

Le modèle sociocogniciel élaboré fédère les dimensions clinique, cognitive, organisationnelle, juridique et technique d'un SADM. Il représente ainsi tous les éléments conceptuels qui permettent d'améliorer la performance cognitive du SADM. Le caractère aléatoire de la prise de décision clinique est par conséquent réduit. Ce modèle est également robuste par rapport aux évolutions technologiques. Dans ces conditions, il est possible de faire l'hypothèse que l'écart entre l'intérêt scientifique d'un SADM et son désintérêt pratique sera réduit de manière significative.

Sur le plan théorique et en référence à l'exemple présenté ci-dessus, les apports de l'approche socio-cognitive pour la conception d'un SADM sont :

- La prise en compte effective des multiples représentations, individuelles et collectives, présentes dans les situations médicales (celles du médecin, du patient et des échanges entre le patient et le médecin);

- Une analyse des interactions par lesquelles les individus produisent leurs conduites sociales et donc agissent sur la décision clinique (cf. la communication patient-médecin);

- Une intégration des cinq critères fondamentaux de la déontologie médicale : indépendance, compétence, information du patient, secret médical, liberté.

Ces apports permettent ainsi la jointure entre la déontologie médicale et la gestion, cette jointure permettant à son tour la gestion sociocognicielle de la responsabilité médicale. Ils permettent également de donner une 
réponse positive à la question posée dans le titre de cet article.

En ce sens, cette conception d'un SADM devient un guide pour l'action. Le modèle sociocogniciel oriente les actions et les relations sociales qui font sens pour les individus (soignants, patient, famille, amis) et les groupes d'individus participant directement ou indirectement à la décision clinique. L'action prédomine sur la représentation, conférant ainsi au SADM un rôle de coordinateur/ intégrateur - "un outil de gestion intermédiaire »- qui permet d'accéder à un monde partagé, plutôt que de résoudre un problème.

Ce monde partagé est d'ailleurs constitué d'une multitude de "smart informations » apparemment infinies, que nous tenons pour acquises (car elles font partie de nos habitudes et de nos réflexes), mais qui doivent être servies à l'ordinateur de manière différenciée et éthique, pour tenir compte des dynamiques humaines d'apprentissage individuelles et collectives.

\section{Conclusion et perspectives}

Dans cet article, partant des défis que pose le phénomène Big data actuel, nous avons proposé un modèle SI d'aide à la décision clinique qui permette une gestion sociocognicielle de la responsabilité médicale. Pour arriver à ce modèle, nous avons expliciter les différentes étapes de la démarche conceptuelle, la finalité de chacune de ces étapes étant d'apporter une contribution à certains questionnements posés par la conception d'un SADM.

La première étape a été de caractériser le Big data sur un plan économique, pour ensuite poser le cadre cognitif et technique des SADM. L'explicitation des différentes étapes cognitives du processus de la décision clinique nous a permis d'appréhender le statut «outil de gestion intermédiaire» d'un SADM lors de l'exercice de la décision clinique.

Le statut d'un SADM étant posé, la deuxième étape a été d'analyser les fondements juridiques du triptyque patient - médecin - SADM/Big Data. Cette analyse nous a conduit à une remise en question strictement physique ou morale de la responsabilité médicale lors de l'utilisation d'un Big Data, à l'avantage d'une réflexion sur les conditions épistémologiques et éthiques de la gestion d'une responsabilité médicale étendue.

Pour mener cette réflexion, la troisième étape a été de proposer une analyse critique des systèmes de connaissances (Ermine, 1996), en référence à la Théorie du Système Général (Le Moigne, 1994). Cette analyse critique a introduit le cadrage théorique de la conception systémique sociocognicielle d'un SADM. Cette conception systémique permet alors une gestion éthique de la communication médecin-patient (cf. l'exemple cidessus).

Le cadrage théorique étant défini, la quatrième étape a été d'expliciter le modèle SADM après avoir présenté l'approche sociocognitive. Le modèle instrumente l'organisation des relations (médecin, patient, SADM) qui se co-construisent et interviennent dans la prise de décision clinique.

Sur un plan pratique, l'approche socio-cognitive à la base du SADM a permis de prendre en compte de manière éthique, les données disparates et volatiles de la communication médecin-patient. Le modèle socio-cogniciel, lui, a permis de « coordonner » (baliser?) et rendre efficace l'organisation des relations (médecin, patient, SADM) qui se co-construisent lors de l'élaboration de la décision clinique. A terme, ce modèle SADM pourrait servir de socle, d'une part, pour la construction d'une base de connaissances, que nous proposons d'appeler « actions et décisions cliniques ", et d'autre part, pour le développement d'un outil de formation à distance destiné, par exemple, à des étudiants en médecine.

Sur un plan conceptuel, la singularité de ce modèle SADM est d'être basée sur une réflexion critique, d'une part, des théories décisionnelles et, d'autre part, des systèmes de connaissances développés par J. L. Ermine. Cette réflexion nous a conduit à adopter une approche méthodologique socio-cognitive, dans la mesure où le modèle SADM proposé est multidimensionnel dans sa conception (dimensions organisation, éthique, cognitive, TIC et décisionnelle). Cette conception prend en compte l'interprétation que les différents acteurs (patient, médecin, soignants, famille, services médico-sociaux) se font de la situation médicale et valorise ainsi les liens entre action et savoir, entre celui qui sait et ce qui est su. Son utilité est de mémoriser et tracer, de manière éthique, les processus, cognitif, organisationnel, décisionnel, sous-jacents à toute activité décisionnelle.

Ce modèle permet aussi de rendre compte du caractère organisant et organisé des liens entre un SADM et les 
pratiques de la décision clinique. En ce sens, le rapport instrumental d'une information et/ou d'une connaissance dans le seul but de décider a été balayé à l'avantage d'un SI orienté «complexité » (Amabile et Caron-Fasan, 2002). Il rend également compte du caractère organisant de la connaissance lors d'une activité décisionnelle, restaurant à ce titre, les dynamiques d'apprentissages individuels et collectifs en lien avec un processus clinique évolutif. Cette « cognition distribuée » rend effective la manière dont des environnements complexes, constitués d'humains et d'artefacts, parviennent à réaliser durablement des tâches complexes dont la décision clinique.

En sciences de l'organisation, l'expérimentation de ce modèle pourrait permettre d'appréhender l'inadéquation du mode de gestion traditionnel issu du modèle Taylorien du travail. Sur un plan pratique de management, la question pourrait porter sur l'émergence d'un nouveau modèle de gestion de l'apprentissage, qui privilégierait les démarches «apprenantes » et responsables, via un artefact SAD Orienté « Connaissances », ce qui compléterait et actualiserait la typologie de Alter (1977).

\section{Bib1iographie}

Aamodt A. et Plaza E. (1994), Case-Based reasoning: foundational Issues, Methodological variations, and System Approaches », AI Communications, IOS Press, Vol. 7, n¹, pp. 39-59

Alter, S. (1977), A Taxonomy of Decision Support Systems, Sloan Management Review, Vol. 19, no1, pp. 39-57

Amabile S. et Caron-Fasan M.L. (2002), Contributions à une ingénierie des systèmes d'information orientée « complexité ", in Rowe F., Faire de la recherche en systèmes d'information, Vuibert FNEGE, pp. 67-78

Banning M. (2006), A review of clinical decision making: models and current research, Journal of Clinical Nursing, 17, pp. 187195

Bernard C. (2013), Introduction à l'étude de la médecine expérimentale, Flammarion, Collection champs classiques, $381 \mathrm{p}$

Berner E.S. (2009), Clinical decision support systems: State of the Art, AHRQ Publication no 09-0069-EF. Rockville, Maryland: Agency for Healthcare Research and Quality. June 2009.

Bousquet F., Barreteau O., Mullon C., Weber J. (2013), Modélisation d'accompagnement : systèmes multi-agents et gestion des ressources renouvelables", dans Rendre possible, Jacques Weber, itinéraire d'un économiste passe-frontières (ouvrage collectif), Editions Quae, Collection indisciplines, pp. 147-155

Buchanan B.G. et Shortliffe E.H. (1984), Rule-Based Expert Systems, the MYCIN experiments of the Stanford Heuristic Programming Project, Ed. Addison-Wesley, $748 \mathrm{p}$

Callon M. (2013), Sociologie des agencements marchands : textes choisis, Presses de l'Ecole des mines, Collection sciences sociales, $482 \mathrm{p}$

Chiapello E. et Gilbert P. (2013), Sociologie des outils de gestion - Introduction à l'analyse sociale de l'instrumentation de gestion, La découverte, Collection Grands Repères Manuels, 260 p

Colin N., Verdier H. (2015), L'âge de la multitude. Entreprendre et gouverner après la révolution numérique (2 édition), Armand Colin, Hors Collection, $304 \mathrm{p}$

Colloc J. (2015), Santé et Big Data: l'Etat et les individus, impuissants face aux pouvoirs des réseaux, http://espacepublique.revues.org/3493, Revue L'Espace Politique [En ligne], 26 | 2015-2, mis en ligne le 26 juillet 2015.

Colloc J. et Sybord C. (2003), A multi-agent approach to involve multiple knowledge models and the case base reasoning approach in decision support systems, In proceedings of the 35th IEEE Southeastern Symposium on System Theory (SSST'03), WVU, Morgantown, 17-18 March 2003, WV, USA, pp. 247-251

Comble P.H., Renaud-Salis J.L., Lagouarde P., Darmoni S. (2010), Etude des Systèmes d'aide à la Décision Médicale - Livrable 2: état des lieux - Partie 1", Projet Cegedim-Activ, Juillet 2010, 46 p

Conseil d'Etat (2015), L'engagement de la responsabilité des hôpitaux publics, Les dossiers thématiques (Mise en ligne le 5 janvier 2015), 14 pages

Darmoni S., Griffon N., Massari P. (2014), Les systèmes d'aide à la décision médicale, Annales des Mines, Réalités industrielles, 2014/4, pp. 47-50 
David A. (2002), Décision, conception et recherche en sciences de gestion, Revue française de gestion n¹39, pp. 173-185

Ehlinger S. (1996), L'approche socio-cognitive de la formation de la stratégie: apports théoriques et méthodologiques, Actes de la Vième Conférence Internationale de Management Stratégique (AIMS), Lille, Mai 1996

Ermine J.L. (1996), Les systèmes de connaissances, Hermès, 160 p

Ermine J.L, Chaillot M., Bigeon P., Charreton B., Malavieille D. (1996), MKSM, méthode pour la gestion des connaissances, Revue Ingénierie des systèmes d'information, AFCET Hermès, Vol. 4, nº 4, pp. 541-575

Falque L., Bougon B. (2009), Pratiques de la décision, Développer ses capacités de discernement, $2^{\circ}$ édition, Dunod, Collection Stratégies et management, $240 \mathrm{p}$

Ferber, J. (1995), Les systèmes multi-agents, vers une intelligence collective », InterEditions, Paris, 522 p

Flottorp S., Oxman A., Krause J., Musila N., Wensing M., Godycki-Cwirko M. et al. (2013), A checklist for identifying determinants of practice: A systematic review and synthesis of frameworks and taxonomies of factors that prevent or enable improvements in healthcare professional practice. Implement Sci 2013 ; 8(1):35

Fernandez A. (2013), Les nouveaux tableaux de bord des managers. Le projet Business Intelligence clé en main (6édition), Eyrolles, 468p

Gallard P.Y. (2014), Aspects juridiques de la décision médicale, Revue Laennec 4/2014, pp. 27-39

Gillet M. et Gillet P. (2013), Les outils du système d'information, facteur clé de succès ou d'échec de l'évolution des organisations : le cas des universités, Revue Gestion et Management public, 2013/3 (Volume 2/nº1), pp. 55-77

Girin J. (1990), Analyse empirique des situations de gestion : éléments de théorie et de méthode, dans Epistémologies et sciences de gestion, coordonné par A.C. Martinet, Economica, 249 p

Gorry G.A. et Scott Morton M.S. (1971), A framework for management information systems, Sloan Management Review, Fall, pp. $55-70$

Gross O et Gagnayre R. (2013), Hypothèse d'un modèle théorique du patient-expert et de l'expertise du patient : processus d'élaboration, Recherches qualitatives 2013, Hors serie; 15: 147-165

Guilbert, J.J. (1992), Comment raisonnent les médecins? : Réflexions sur la formation médicale, Ed. Médecine et Hygiène, Genève, Suisse, $97 \mathrm{p}$

Haji Safar S. (2012), Information du patient : recommandation de bonne pratique de l'HAS de mai 2012 et jurisprudence de référence, Revue Droit, Déontologie \& Soin, Volume 12, Issue 4, December 2012, pp. 445-459

Hoerni B. (2000), Ethique et déontologie médicale, $2^{\circ}$ édition, Elsevier Masson, Collection Abrégés, 115 p

Hoerni B. (2008), L'évolution des échanges patient-soignant, Imothep éditions, 288 p

Intel (2012), Big Data Analytics. Intel's Manager Survey on how organizations are using Big Data, Août 2012, 27 p

Kahneman, D., and Klein, G. (2009), Conditions for intuitive expertise: A failure to disagree, American Pschologist (64:6), pp. 515-526.

Kessous E. (2012), L'attention au monde. Sociologie des données personnelles à l'ère numérique, Armand Colin/ Recherches, $320 \mathrm{p}$

Klein G. A., Orasanu J., Calderwood R. et Zsambok C. E. (Eds., 1993). Decision making in action: Models and methods, Norwood, NJ: Ablex Publishing Corporation

Klein, G. A. (1999 (1 $1^{\text {ière }}$ ed. en 1998)), Sources of power: How people make decisions, Cambridge, MA: MIT Press, 352 p

Langton C. (1997), Et si les informaticiens découvraient la vie ? Courrier International n 360, 25 sept. - $1^{\circ}$ oct. 1997

Lauriol J. (1998), La décision stratégique en action : une perspective socio-cognitive, L'Harmattan, $284 \mathrm{p}$

Lebraty J.F. (2006), Les systèmes décisionnels, Dans Encyclopédie de l'informatique et des systèmes d'information sous la direction de J. Akoka et I. Comyn-Wattiau, Vuibert, pp. 1338 - 1349 
Le Moigne, J.L. (1974), Les systèmes de décision dans les organisations, Presses Universitaires de France, $244 \mathrm{p}$

Le Moigne, J.L. (1993 (1 1 ière ed. en 1990), La modélisation des systèmes complexes, Dunod, Collection Sciences humaines, AFCET Systèmes, $178 \mathrm{p}$

Le Moigne J.L. (1994 (1 $1^{\text {ière }}$ ed. en 1977)), La théorie du système général (4º́dition), Paris, PUF, 338 p

Le Moigne J.L. et Amabile S. (2006), Épistémologie des systèmes d'information, in Akoka J, et Comyn-Wattiau I (éds.), Encyclopédie de l'informatique et des systèmes d'information, Vuibert, Paris, pp. 211-226.

Marr B. (2015), Big Data: Using SMART Big data, Analytics and Metrics To Make Better Decisions and Improve Performance, John Wiley \& Sons, $256 \mathrm{p}$

Martinet A.C. (2014), La multitude et la fluidité : implications pour la stratégie, Revue Française de Gestion, $2014 / 7$ (n²44), pp. 73-91

Michit R. (1998), Une méthode d'explicitation de la structure du processus décisionnel des individus et des groupes : l'entretien psycho-cognitif », Revue Communication et organisation, 14/ 1998, pp. 232-253

Montebello M. (1976), Efficacité de l'entreprise : analyse et perspectives, Thèse de doctorat d'Etat ès Sciences de Gestion, Université d'Aix-Marseille III, octobre.

Murakami Wood D. et Ball K. (2013), Brandscapes of control? Surveillance, marketing and the co-construction of subjectivity and space in neo-liberal capitalism, Marketing Theory, 13(1), pp. 47-67

Myers, J.D., Pople, H.E., Miller, R.A. (1982), CADUCEUS : a computerized Diagnostic Consultation System in Internal Medicine, Pittsburgh, USA, Decision system Laboratory

Newell A., Simon H. (1972) Human problem solving, Prentice-Hall, Englewood Clifs, 920 p

Noucher M. (2009), La donnée géographique aux frontières des organisations : approche socio-cognitive et systémique de son appropriation, Thèse, Avril 2009

Olry-Louis I. (2011), Interactions à visée d'apprentissage et différences individuelles, Chapitre 2 de Interactions dans le groupe et apprentissages, coordonnée par Hugon M. A et Le Cunff C., Nanterre, Presses Universitaires de Paris Ouest, pp. 31-41

Pelaccia T., Tardif J., Triby E., Ammirati C., Bertrand C., Charlin B. (2011), Comment les médecins raisonnent-ils pour poser des diagnostics et prendre des décisions thérapeutiques? Les enjeux en médecine d'urgence, Annales françaises de médecine d'urgence, Vol.1, Issue 1, pp. 77-84

Poiraud P. (2013), Ne manque-t-il pas un V au Big Data? Le club des Echos, le 31/01/2013

Pople, H.E., Myers, J.D., Miller, R.A (1975), DIALOG : a model of diagnosis logic for internal medicine, in proceedings of $4^{\text {th }}$ International Joint Conference for Artificial Intelligence, IJCAI, Tbillissi, pp. 74-87

Ramonet I. (2015), L’Empire de la surveillance, Éditions Galilée, 192 p

Rennel G. et Shortliffe E.H. (1987), Advanced Computing for Medicine, Sci. Amer., 257, pp. 154-161

Rouvroy A. et Berns T. (2013), Gouvernementalité algorithmique et perspectives d'émancipation : le disparate comme condition d'individuation par la relation?, Revue Réseaux 2013/1 (n¹77), pp. 163-196

Rowe, F. (2007), Systèmes d'information : variations philosophiques sur une proposition de définition ", dans Connaissance et management, ouvrage dédié à R.Reix et coordonné par P.L. Dubois et Y.Dupuy, pp. 165-173

Sargos, P. (1996), La responsabilité civile en matière d'exercice médical pluridisciplinaire, Revue Médecine et Droit, n¹7, p.17-20

Séroussi B. et Bouaud J. (2014), Systèmes informatiques d'aide à la décision en médecine : panorama des approches utilisant les données et les connaissances, Revue Pratique neurologqiue -FMC, 2014, 5(4), pp. 303-316

SFMG (1996), Documents de recherches en médecine générale, Dictionnaire des résultats de consultation en médecine générale, Société Française de Médecine Générale, Numéro spécial 47-48, décembre 1996, 368 p

Shen Y., Colloc J., Jacquet-Andrieu. A. (2014), Elaboration d'ontologies médicales à partir de textes ou corpus via un méta- 
modèle, in proceedings AMINA'2014, pp. 111-116

Simon H.A. (1983), Administration et processus de décision, Economica, $3^{\circ}$ édition, $322 \mathrm{p}$

Sybord C. (1992), Méthode de développement propre aux systèmes experts : la méthode Q4, Thèse, Université Jean Moulin Lyon 3, France, Février 1992

Sybord C. (2000), Système d'aide à la décision et responsabilité médicale : vers une gestion de la responsabilité médicale, Communication des XV Journées nationales des IAE, Bayonne - Biarritz, 6 - 8 Septembre 2000

Sybord C. (2007), Intelligence économique et situations de décision : proposition d'un modèle socio-cogniciel, Publications du $5^{\circ}$ colloque international VSST'2007 (Veille Stratégique, Scientifique et Technologique), Marrakech (Maroc), 21 - 25 Octobre 2007

Sybord, C. (2013), Big Data et système d'aide à la décision : les apports d'une approche socio-cognitive pour la «Little Knowledge ", $18^{\circ}$ Colloque européen de l'Association Information et Management (A.I.M.), Thème «BIG DATA : les défis de la gestion des grands volumes de données », (F) Lyon, 22-24 Mai 2013

Sybord, C., Colloc, J. (1997), A cognitive approach of the decision process to develop health decision support systems, ECIS'97, 5th European Conference on Information Systems, Cork, Ireland, 1997, pp. 396-412

Thoër C. (2013), Internet : un facteur de transformation de la relation médecin-patient ? Revue Internationale Communication sociale et publique, $\mathrm{n}^{\circ} 10$, pp. 1-24

Vayre J.S. (2013), Le big data et la relation client. Quand les traces numériques organisent l'échange marchand, $12^{\circ}$ Journées Normandes de Recherches sur la Consommation : Société et Consommation, Novembre 2013, pp. 1-20

Vidal P. et Lacroux F. (2000), L'évolution des systèmes d'aide à la décision: du choix en situation structurée à l'intermédiation en situation complexe, Revue Systèmes d'Information et Management, Vol.5, n³, pp. 51-70

Villey, R. (1982), Déontologie médicale, Masson, 106 p

Zolynski C. (2015), Big data : pour une éthique des données, Revue i2D, 2015/2 (Volume 52), pp. 25-26

Title: Big Data and design of clinical decision support system: Towards a management of the medical accountability?

\begin{abstract}
Given all the challenges that Big Data is facing for storage, analysis and protection of personal health data, assessing a Clinical Decision Support System (CDSS) has become critical for both physicians and patients. In this context, the article covers how to design a CDSS and the conditions under which it can be integrated into the clinical workflow of medical practice. After defining the economic impact of Big Data, the first section will introduce the technical and cognitive framework of CDSS. Once the positioning of a CDSS is defined, the second section will analyze the legal foundations of the three-fold framework patient - physician - CDSS/Big Data. The third section is a critical analysis of the knowledge based system referring to the theory of the general system. This critical analysis introduces the theoretical framework of the CDSS design. The fourth section present the sociocogniciel model of a CDSS. This model surrounds the medical accountability.
\end{abstract}

Key words: Big Data, Clinical decision, Clinical Decision Support System, Medical accountability, Complex system modelling, Knowledge-based systems, Sociocognitive approach.

\title{
Coordonnées Auteure
}

Université Lumière Lyon 2

Laboratoire COACTIS EA 4161

ISH 14/16 avenue Berthelot

F-69363 Lyon Cedex 07

Tél. : +33478697610

E-mail: christine.sybord@,univ-lyon2.fr 